\title{
Transient Left Eyelid Incompetence After Mandibular Block Anesthesia: A Case Report
}

Andri Hardianto ${ }^{1}$ and Wilson Wijaya ${ }^{2 *}$

*Correspondence: wilson_wijaya@live.com

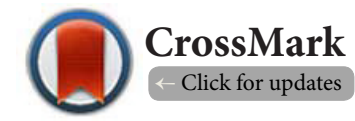

'Department of Oral and Maxillofacial Surgery, Faculty of Dentistry, Padjadjaran University, Bandung 40132.

${ }^{2}$ Dental Unit, Karitas Catholic Hospital, South West Sumba 87255.

\begin{abstract}
Background: Inferior alveolar nerve block prior to lower posterior tooth extraction is often done in dentistry. Rarely, clinician may pull aniatrogenic error by inaccurately positioning the tip of the syringe. Anesthetic solution may damage the ganglion of 7 th cranial nerve, resulting in Facial Palsy, including eyelid incompetence of the side being anesthesized.

Objectives: The purpose of the case is to report eyelid incompetence as one the less common side-effect of inferior alveolar nerve block.

Case: A 32-year-old woman came to the Karitas Catholic Hospital Dental Unit for second left molar extraction with inferior alveolar nerve block as anesthesia. As the anesthesia came into effect, the left eyelid was unable to close at the same time.

Management: Sterile gauze with saline was put over her left eye to avoid dryness and an anti-inflammatory drug was prescribed.

Conclusion: Dentists have to prepare for every side-effect of inferior alveolar nerve block, including eyelid incompetence.

Keywords: Inferior alveolar nerve block, eyelid incompetence, facial palsy
\end{abstract}

\section{Introduction}

Lagopthalmos is inability to close the eyelid, which is one of the symptoms of Facial Palsy (FP), ranging from mild to complete form [1]. This condition may be caused by a lot of factors, such as trauma, infection, and tumor, including iatrogenic trauma by a clinician in the form of a nerve lesion, ranging from concussion (neuropraxia) to complete separation of nerve body (neurotmesis) [2].

Dental anesthetics in the form of Inferior Alveolar Nerve Block (IANB) is one of the most frequent causes for nerve lesions, as the target of anesthetic injection lies closely to the parotid salivary gland, which encapsulates the $7^{\text {th }}$ cranial nerve's ganglion $[3,4]$. This article aims to present a case of iatrogenic-induced facial palsy due to mandibular block prior to tooth extraction and to review the pathophysiology of lagopthalmos and explain its management.

\section{Case presentation}

A 32-year-old woman came to the dental unit in Karitas Hospi- tal, South West Sumba, East Nusa Tenggara, for second lower left molar extraction with a necrotic pulp. She had no medical compromise recorded before. She had her first lower right molar extracted using inferior alveolar nerve block 2 years prior with no complication. A left inferior alveolar nerve block using $2 \mathrm{ml}$ of $2 \%$ lidocaine was administered. After 5 minutes, the patient felt numbnesson her left lip and cheek. At the same time, she complained of the inability to fully close her left eyelid (Figure 1).

When the patient complained of the inability to close her eyelid, clinical examination was immediately done and was found that the patient had no other facial motoric disturbance other than eyelid incompetence. Dental extraction was postponed to another date, the patient was given sterile gauzes soaked in saline solution to cover her left eye, prescribed artificial tears and anti-inflammatory drops, and was scheduled to come back on the next day.

The patient came the next day, stated that her left eye was able to close completely at the same timeas the numb sensation on her left lip subsided. 


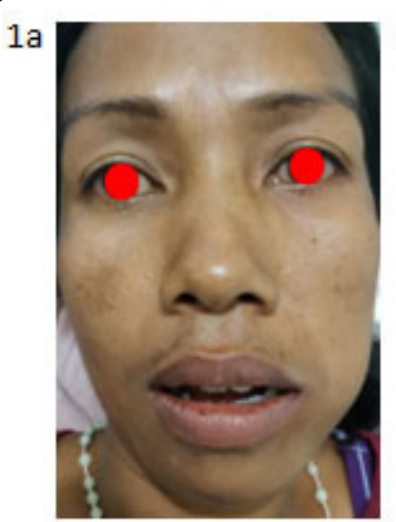

$1 b$

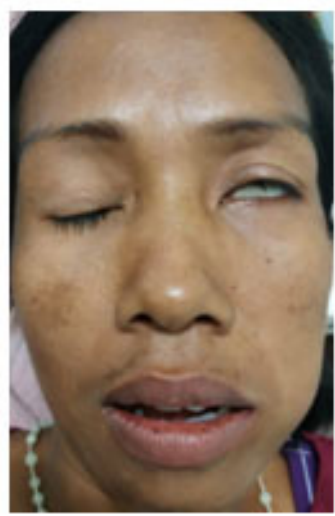

Figure 1. (a)Clinical examination of fully opened eyelid. (b). Clinical examination of one-sided lagopthalmos.

The patient could not be referred to a neurologist due to the absence of neurologistsin the island and the patient could not afford transportation to the nearest neurologist.

\section{Case management}

Anesthesia is one of the most common procedures done in dentistry, mostly used for restorative dentistry, esthetic dentistry, and many more, including oral surgery for pain relief, pre-operative pain, intra-operative pain, post-operative pain, and diagnostics [2]. There are a number of anesthesia techniques, and one of the most common techniques is inferior alveolar nerve block (IANB), that works by filling the lingula on the mandible ramus with anesthetic solution, commonly $2 \%$ lidocaine combined with 1:100.000 epinephrine using a syringe. This procedure will induce numbness on the ipsilateral sensory inferior alveolar nerve, thus the patient will have none or little pain sensation when manipulated by the operator [5]. Its uses aside, IANB also has its complications, from the mildest form such as loss or altered sense of taste (dysesthesia), trauma to blood vessels and nerves, the latter causing disruption of speech, face expression, and eyelid function [3].

In this case, the patient needed IANB for the extraction procedure of second left lower molar with the diagnosis of necrotic pulp. IANB was done with the classic indirect technique with 2\% lidocaine with 1:100.000 epinephrine solution.

When the anesthetic solution for IANB was administered, the patient started feeling numbness in her left side of lip. This is one of the signs that shows successful IANB. However,at the same time, her left eyelid was not able to close down, one of the symptoms of Facial Palsy. The patient's left eyelid incompetence was caused by a temporary injury to the left orbicularis oculi nerve branch of the $7^{\text {th }}$ cranial nerve's temporal nerve branch, due to trauma by the anesthetic solution $[7,8]$ (Figure 2).

Damage to the temporal branch of the $7^{\text {th }}$ cranial nerve (CN VII) is mostly caused by trauma, such as surgery, and blunt or sharp external force [7], affecting the orbicularis oculi muscle, commonly causing neuropraxia (concussion), causing the ipsilateral eyelid to be unable to close completely [9]. Steenen, et al reviewed literatures that showed 131 cases of unusual ocular complications caused by intra oral injection from 1936 to 2011 [8]. Even though it was rarely encountered, dental procedure such as IANB can cause this condition. Because of the proximity of the target for IANB anesthesia and CN VII's ganglion, which is located inside the parotid gland [4], when the tip of the anesthesia needle is placed too posteriorly or there is parotid gland anatomy variation, the anesthesia solution may damage the ganglion, causing complications such as eyelid dysfunction, as stated by Alamanos et al in 2016 [10].

One of the explainable reasons for this is the position of the syringe tip that is too posteriorly placed, thus injecting the anesthetic solution into the parotid salivary gland. In this case, the anesthetic solution temporarily damaged the orbicularis oculi branch of $7^{\text {th }}$ cranial nerve's temporal branch.

Facial Palsy (FP) can be caused by a lot of factors, such as iatrogenic, trauma, tumor, and even systemic diseases, like a stroke. For those reasons, the cause of FP has to be quickly determined and distinguished from other causes [2]. In this case, the patient has no previous medical history, and lagopthalmos was found at the same time as the onset ofnumbness on her lip and tongue. It was probable that there was an anatomic variation of the parotid gland, similar as stated by Kempster et al [11].

Eyelid incompetence can cause Dryness Eye Disease (DED) from dryness caused bya constantly opened eyelid. To prevent this, the patient is given sterile gauze soaked in saline solution and artificial tears to avoid dryness, and anti-inflammatory drugs to prevent keratitis [12] (Figure 3).

The patient is told to come to the clinic the next day to check up on her eyelid, and stated that her eye can close completely when the numbing on her lip and tongue faded, around 2 hours after the onset of FP.

Verbal consent was obtained from the patient on the second 


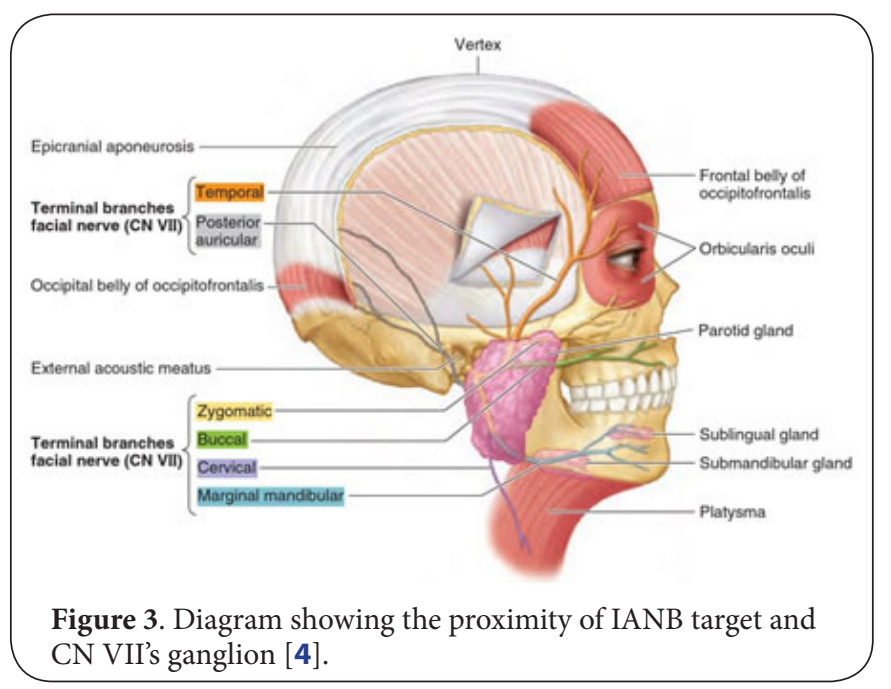

day of her visit for the use of her photo for this case report.

\section{Conclusion}

Facial Palsy is one of the lesser known side effects of an IANB procedure, one of the symptoms being the inability to close the eyelid. Clinicians should have the ability to diagnose, find the cause of FP, and treat it, as it can happen to any patient, mainly patients that need IANB for their routine dental treatment. More importantly, clinicians should strive to prevent iatrogenic $\mathrm{FP}$, avoiding lowering the patient's quality of life instead of increasing it according to the patient's expectations.

\section{Competing interests}

The authors declare that they have no competing interests.

\section{Authors' contributions}

\begin{tabular}{|l|c|c|}
\hline Authors' contributions & WW & AH \\
\hline Research concept and design & $\sqrt{ }$ & $\sqrt{ }$ \\
\hline Collection and/or assembly of data & $\sqrt{ }$ & -- \\
\hline Data analysis and interpretation & $\sqrt{ }$ & $\sqrt{ }$ \\
\hline Writing the article & $\sqrt{ }$ & -- \\
\hline Critical revision of the article & -- & $\sqrt{ }$ \\
\hline Final approval of article & -- & $\sqrt{ }$ \\
\hline Statistical analysis & $\sqrt{ }$ & -- \\
\hline
\end{tabular}

\section{Acknowledgments}

Acknowledgements to Fidelis Jacklyn Adella as grammar and thesaurus proof-reader.

\section{Publication history}

Editor: Giuseppina Nocca, Catholic University of Sacred Heart, Italy. Received: 21-Jan-2021 Revised: 27-Feb-2021

Accepted: 02-Mar-2021 Published: 21-Mar-2021
2. Ilea A, Cristea A, Tărmure V, Trombitaș VE, Câmpian RS, Albu S. Management of patients with facial paralysis in the dental office: A brief review of the literature and case report. Quintessence Int Berl Ger 1985. 2014 Jan;45(1):75-86.

3. Ngeow WC, Shim CK, Chai WL. Transient loss of power of accommodation in 1 eye following inferior alveolar nerve block: report of 2 cases. J Can Dent Assoc. 2006 Dec;72(10):927-31.

4. Moore KL, Dalley II AF, Agur AMR. Clinically Oriented Anatomy. 7th ed.

5. Hupp JR, Ellis III E, Tucker MR. Contemporary Oral and Maxillofacial Surgery. 6th ed. Elsevier;

6. Khoury J, Townsend G. Neural Blockade Anaesthesia of the Mandibular Nerve and Its Terminal Branches: Rationale for Different Anaesthetic Techniques Including Their Advantages and Disadvantages. Anesthesiol Res Pract. 2011 May 25;2011:307423.

7. Wolber P, Volk GF, Horstmann L, Finkensieper M, Shabli S, Wittekindt C, et al. Patient's perspective on long-term complications after superficial parotidectomy for benign lesions: Prospective analysis of a 2-year followup. Clin Otolaryngol. 2018;43(4):1073-9.

8. Steenen SA, Dubois L, Saeed P, de Lange J. Ophthalmologic complications after intraoral local anesthesia: case report and review of literature. Oral Surg Oral Med Oral Pathol Oral Radiol. 2012 Jun;113(6):e1-5.

9. VanderWerf F, Reits D, Smit AE, Metselaar M. Blink Recovery in Patients with Bell's Palsy: A Neurophysiological and Behavioral Longitudinal Study. Invest Ophthalmol Vis Sci. 2007 Jan 1;48(1):203-13.

10. Alamanos C, Raab P, Gamulescu A, Behr M. Ophthalmologic complications after administration of local anesthesia in dentistry: a systematic review. Oral Surg Oral Med Oral Pathol Oral Radiol. 2016 Mar 1;121(3):e39-50.

11. Kempster C, Ghabriel M, Kaidonis G, Townsend G. An unusual ocular complication following dental anaesthesia: case report. Aust Dent J. 2016;61(3):374-80.

12. Alves M, Fonseca EC, Alves MF, Malki LT, Arruda GV, Reinach PS, et al. Dry Eye Disease Treatment: A Systematic Review of Published Trials and a Critical Appraisal of Therapeutic Strategies. Ocul Surf. 2013 Jul 1;11(3):181-92.

\section{Citation:}

Hardianto A and Wijaya W. Transient Left Eyelid Incompetence After Mandibular Block Anesthesia: A Case Report. Oral Biol Dent. 2021; 9:1.

http://dx.doi.org/10.7243/2053-5775-9-1

\section{References}

1. Lawrence SD, Morris CL. Lagophthalmos Evaluation and Treatment [Internet]. Available from: https://www.aao.org/eyenet/article/ lagophthalmos-evaluation-treatment 